\title{
The effect of ischemia on the peripheral nerve action potential in man and its relation to somatosensory magnitude coding
}

WILLIAM R. UTTAL

UNIVERSITY OF MICHIGAN

The amplitude and latency fluctuations of the compound action potential of the ulnar nerve were recorded percutaneously as a function of the length of time the arm was kept ischemic by means of an inflated sphygmomanometer cuff. Changes in latency and the amplitude action potential were measured for cuff positions above and below the recording electrode. The amplitude fluctuations were shown to be related to corresponding changes in the sensory threshold which has been determined in an earlier study. By manipulating the position of the cuff it was possible to determine that the amplitude fluctuations were caused by a reduced number of nerve action potentials rather than a change in the individual axonal action potential amplitude.

One of the major problems in psychophysics has been the determination of the code of the neural message which conveys information about sensory magnitude from the periphery to the central nervous system. In two earlier studies (Uttal, 1959, 1960), Dawson and Scott's (1949) method for recording nerve action potentials through the human skin had been developed and used simultaneously with magnitude estimates to demonstrate the relationship between these psychophysical and neurophysiological measures. Some clues were thus provided to the nature of the code. Prior to this work, the results of a study (Uttal, 1958) had been reported on the fluctuations of the psychophysical threshold as a function of time under ischemic conditions created by an inflated sphygmomanometer cuff. It is the purpose of this present study to combine the techniques of percutaneous nerve action potential recording and ischemic analysis (a technique first reported by Magladery, McDougall, \& Stoll (1950)) and to compare the time course of the nerve action potentials to the psychophysical results.

It is also necessary to distinguish between two alternative explanations for the variation in nerve action potential amplitude which resulted from the ischemia. This was accomplished by performing the experiment with the sphygmomanometer cuff in two different positions. The results of this study add further support to the conclusion that sensory magnitudes in the somatosensory system appear to be encoded in the periphery by a simple count of the number of axonal responses.

\footnotetext{
Subjects

METHOD

Three male undergraduate students from the Uni-
}

versity of Michigan served as paid Ss in this experiment. None had a history of cardiac and neurological disturbances of any kind.

\section{Procedure}

The apparatus used in this experiment has been described in detail in the earlier reports (Uttal, 1959, 1960). Constant current, voltage isolated electrical pulses were conveyed to a stimulating electrode

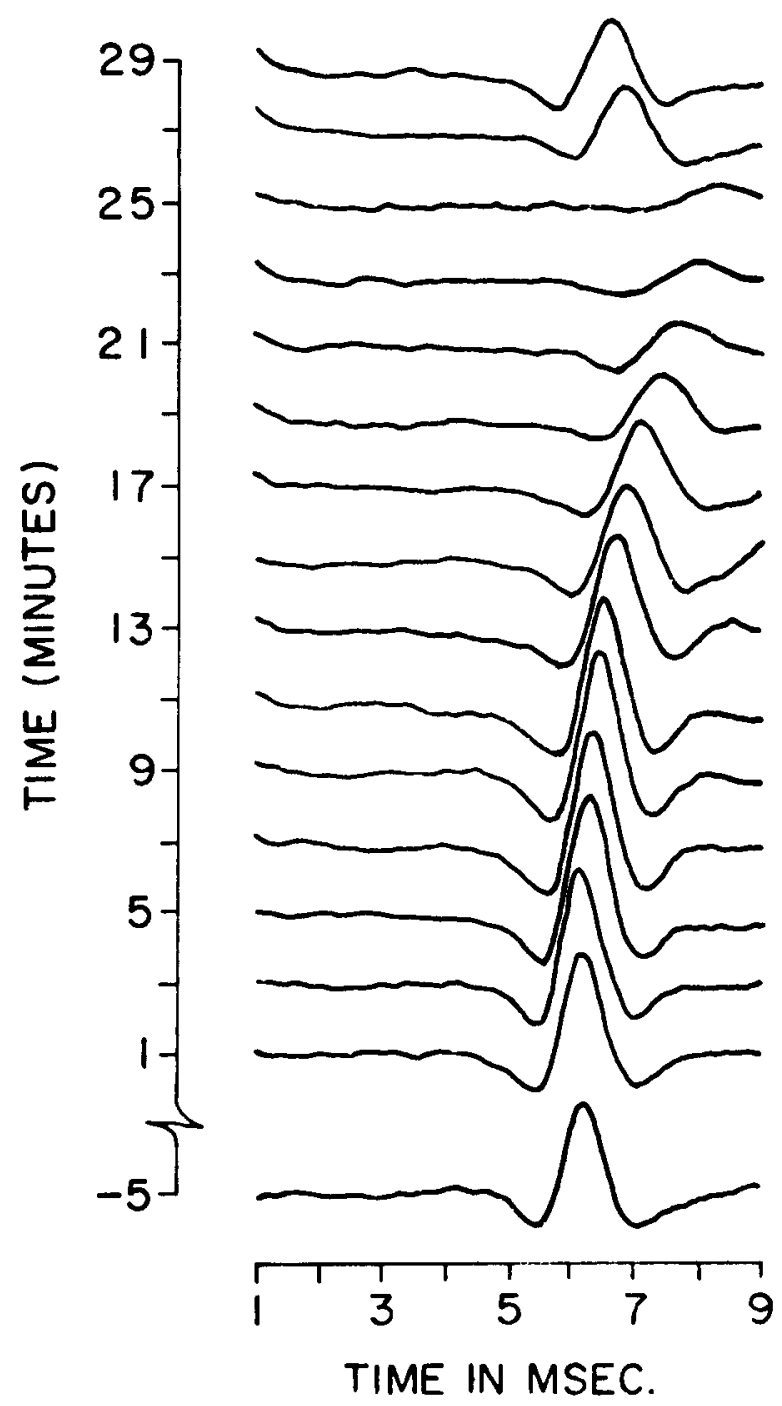

Fig. 1. Tracings of the change in nerve action potential amplitude in a typical run. (Only every other record has been traced.) 
F. S

O CUFF ON LOWER ARM

- CUFF ON UPPER ARM
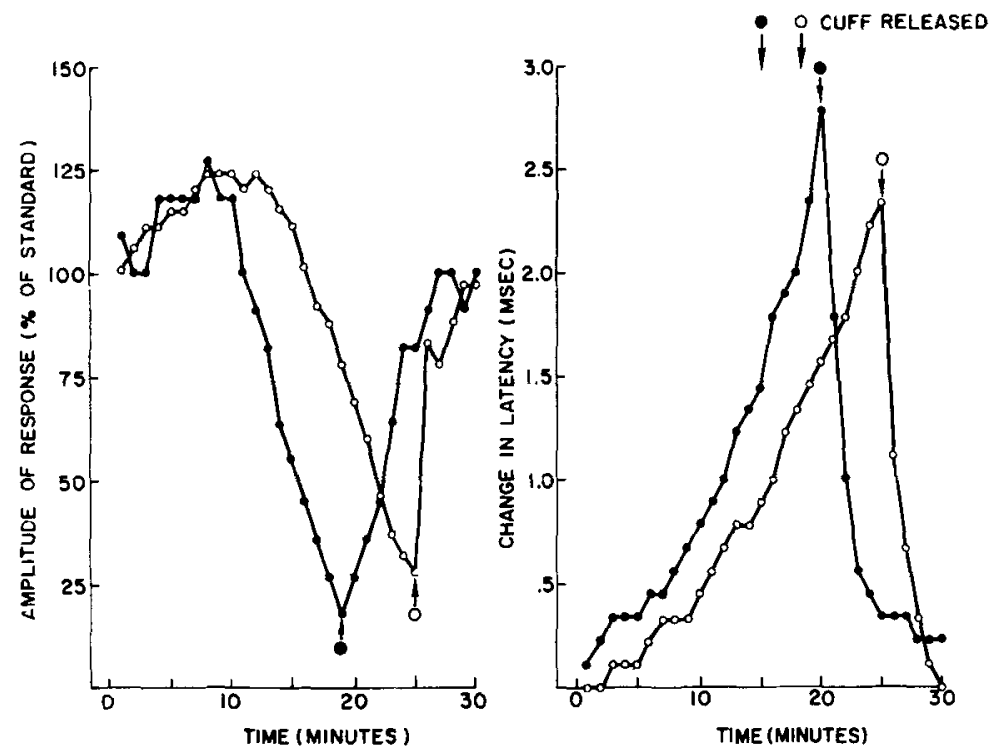

Fig. 2. The effect of ischemia on the nerve action potential as a function of time for the two-cuff positions. Data for F.S. placed over a superficial portion of the ulnar nerve at the wrist. A plastic bar supporting the stimulating electrodes also supported a large ground electrode which helped to reduce stimulus artifact. The recording electrodes were placed above another superficial portion of the ulnar nerve just above the elbow. The electrodes, in each case, were hemispherical stainless steel buttons approximately 5/16 in. in diameter.

Preparation of the skin and accurate location of the electrodes is a critical part of this technique. The location of the ulnar nerve on the left arm was determined by probing for a spot which produced a maximal twitch of the little finger with minimal test stimulus. These two areas were lightly sandpapered, scrubbed with electrode jelly and then washed with soap and water and dried. The electrodes were lightly coated with electrode jelly and then strapped to the preparation locations with rubber bands. The S's hand was held in place by a large weighted rubber strap placed across the palm to minimize movements of the electrode relative to the nerve.

Ischemia was produced by the inflation of a sphygmomanometer cuff to a pressure of $180 \mathrm{~mm}$ of mercury. The cuff was located in two different places in subsequent sessions with the Ss. The first position was on the upper arm above the recording electrodes. In this position the ulnar nerve was ischemic both at the recording and stimulating electrodes. The second position was on the upper forearm, thus creating an ischemic condition only at the portion of the nerve at the stimulating electrode and the other portions of the arm below cuff. The pallor, cyanosis, and reduced temperature of the arm was in each case only below the pressure cuff and was not present in the upper arm when the cuff was below the elbow. After a series of calibration pictures taken at 5, 3, and $1 \mathrm{~min}$. before the inflation, the cuff was inflated and records taken of the nerve action potential response to a $4 \mathrm{~mA}$, $5 \mathrm{msec}$. electrical shock each minute for about $25 \mathrm{~min}$. The length of time of each session was dependent upon the time taken to reduce the nerve impulse to an immeasurably small amplitude. In most cases this was 25 min., but as our records show, one session was terminated at 20 and one at $30 \mathrm{~min}$. Records were also taken at 1-min. intervals during the first $5 \mathrm{~min}$. of the recovery period. The amplitudes and latency of the response were then measured by hand and plotted as shown in our figures. Because of the nature of the physiological manipulation, the possibility of a cumulative deficit and the relatively invariant nature of the data, each $S$ was used only once in each condition.

Display of the action potentials was done by means of a Tektronix 564 storage oscilloscope. The stored pictures were then photographed with a Polaroid type camera.

\section{RESULTS}

Figure 1 is a set of tracings from Polaroid photographs of a typical series of even-numbered nerve action potentials from E.S. during a session in which the sphygmomanometer cuff has been placed on the upper arm. Owing to the positioning of the oscilloscope camera, a small portion of the early and late parts of each trace has been cut off. Thus, we are not able to see the stimulus artifact at the initial portion of the sweep or that portion of the sweep after the response.

Figures 2, 3, and 4 show the results of the experi- 
ment for each S. The data are plotted for both ischemic conditions (cuff on upper arm and cuff on upper forearm) superimposed on the same graph and normalized by taking percentages of the average value of the three calibration records taken at $-5,-3$, and -1 min. The curves are similar for all Ss and relatively monotonic from point to point. Latencies have been plotted as the change in milliseconds from the average latency of the three calibration records.

The shape of the amplitude function can be seen to be a complex function in which a 12- to 15-min. period of supernormality (not reported by Magladery, McDougall, \& Stoll (1950)) is followed by a monotonically decreasing period until the nerve amplitude diminishes to an immeasurably small value. Following release of the cuff the action potential amplitude rapidly recovers to its original value, usually in less than $5 \mathrm{~min}$. One of the most interesting results of this experiment is the lack of differences between the amplitude functions of the responses recorded above as opposed to those recorded below the inflated cuff. Similarly the latencies do not show any meaningful difference between the two conditions.

\section{DISCUSSIOH}

The significance of these data lie in relationship to the psychophysical function described in the earlier paper (Uttal, 1959). Figure 5 is an idealized interpretation of the earlier results and the present results of the sessions with the cuff on the upper arm. Each critical point (i.e., minimum or maximum) is indicated at the average value of the appropriate sessions. It can be seen that the average point of maximal sensitivity of the psychophysical threshold occurs approximately $2 \mathrm{~min}$. after the average time at which the nerve amplitude begins to decrease away from the peak of its early supernormal period. Also, it can be seen that the psychophysical threshold elevates enormously as the nerve action potential amplitude decreases toward an immeasurably small value. It can be further observed that the rapid recovery of the nerve action potential and the psychophysical threshold after the deflation of the cuff are concurrent. It is important to remember that the amplitude of the compound action potential represents the response of nearly the total population of nerve fibers of the ulnar nerve to a strong stimulus. On the other hand the psychophysical threshold curve is representative of a very small population of low threshold neuronal elements. The discrepancies between corresponding points of the psychophysical threshold and the nerve response amplitude may thus be explained in terms of this difference in population.

The question remains of the origin of the amplitude change. Two possibilities could create such a dynamic function. In the first, the decrease in oxygen supply could alter the metabolism of the nerve so that the amplitude of each individual axonal potential varied as a function of the time under ischemic conditions. On the other hand, we are dealing with a compound action potential, which would also vary in amplitude if the number of active nerve fibers changed during the time course of the experiment.

It was to determine which one of these possibilities was the actual cause of the systematic change in nerve action potential amplitude that we repeated the ischemic experiment with the cuff placed below the recording electrode but above the stimulating electrode rather than having both electrodes in the ischemic region. We would expect that in the former case, since the upper part of the ulnar nerve is getting
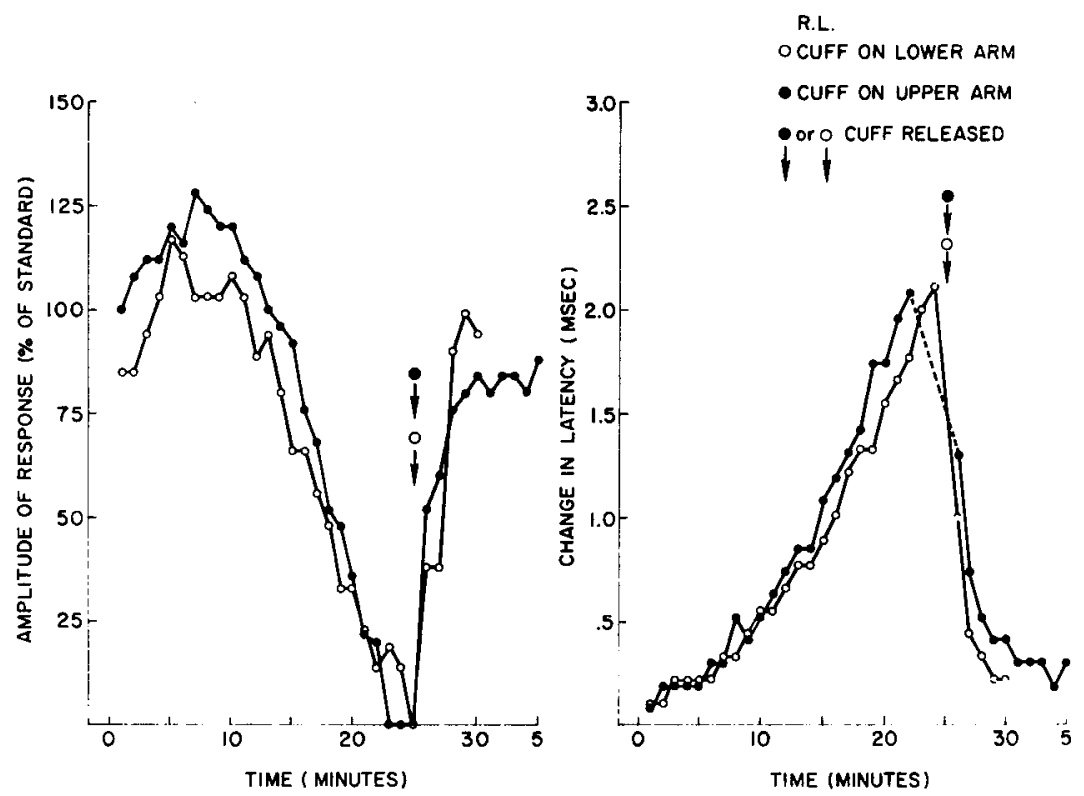

Fig. 3. Data for R.L. 


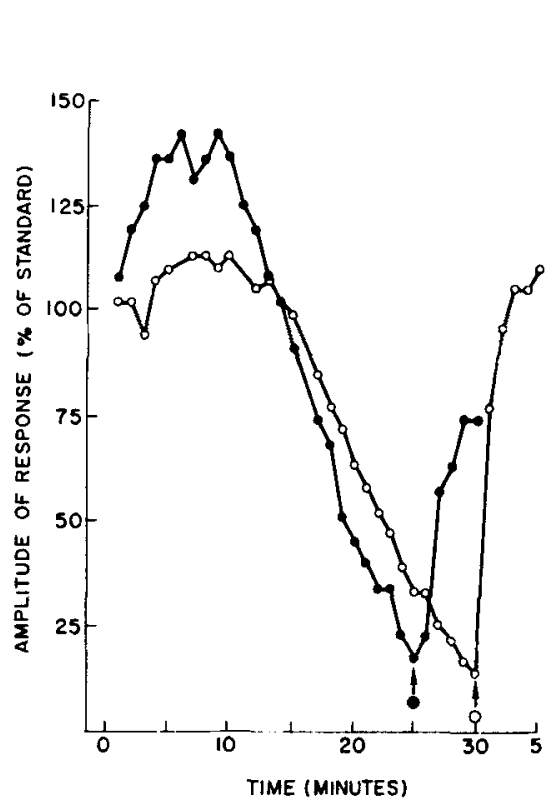

an adequate supply of oxygen from its unoccluded collateral circulation, that the amplitude would not change if the effect was due to a metabolic deficit reducing the individual axonal action potential amplitude. However, if a reduced number of action potentials were being transmitted up the ulnar nerve, such a reduction should also show a diminution in the compound nerve action potential amplitude above the cuff. The results are clear cut, since the reduction in amplitude occurs equally in both conditions.

Indeed, it is somewhat surprising that the difference between the results due to the two positions is so slight. It might well have been expected that

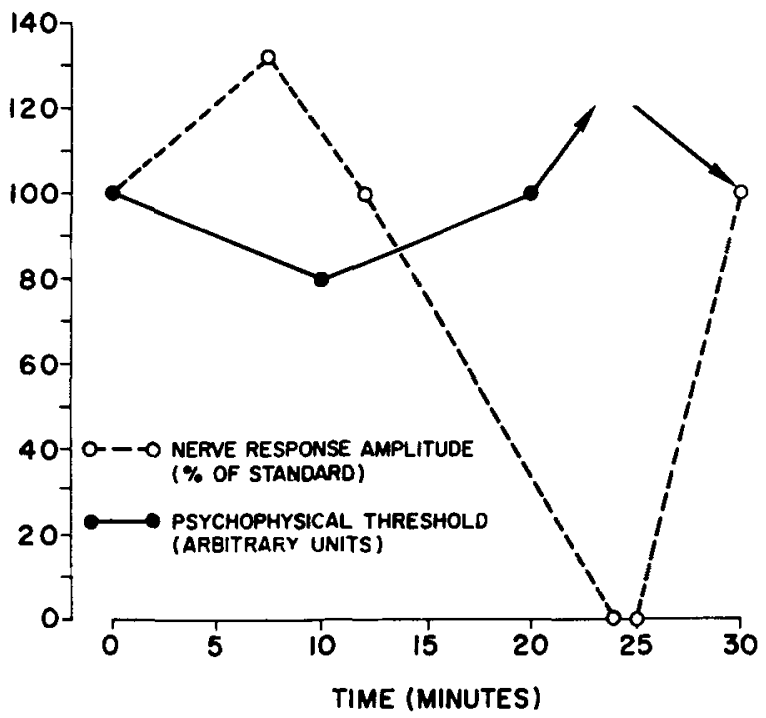

Fig. 5. An idealized comparison of the results of this experiment and the results of the earlier study in which the psychophysical threshold was measured as a function of ischemia.
CUFF ON LOWER ARM

- CUFF ON UPPER ARM

- or O CUFF RELEASEO

i $i$ i

Fig. 4. Data for E.S. although the number of fibers firing would be the dominant effect, there should be some alteration of the response as a function of the condition of the nerve in which the signals are being conducted. The very slight differences between the two conditions for all Ss do suggest that almost all of the variability is due to changes in the thresholds. Such a result strengthens the argument put forth in our earlier papers that it is the number of pooled action potentials (as reflected in the amplitude of the compound action potential) which is the correlate of sensory magnitude since they are herein shown to be also related to the threshold-one of the most critical points of subjective magnitude. As shown in these earlier papers, the interval between successive responses serves only to provide more responses to be counted within a critical counting interval, and the actual timing of the responses is an irrelevant cue.

\section{References}

Dawson, G. D., \& Scott, J. W. The recording of nerve action potentials through skin in man. J. Neurol. Neurosurg. Psychiat., 1949, 12, 259-267.

Magladery, J. W., McDougall, D. B., Jr., \& Stoll, J. Electrophysiological studies of nerve and reflex activity in normal man. II. The effects of peripheral ischemia. Bull. Johns Hopkins Hospital, 1950, 86, 291-312.

Uttal, W. R. Cutaneous sensitivity to electrical pulse stimuli. J. comp. physiol. Psychol., 1958, 51, 549-554.

Uttal, W. R. A comparison of neural and psychophysical responses in the somesthetic system. J. comp. physiol. Psychol., 1959, 52, 485-490.

Uttal, W. R. The three-stimulus problem: a further comparison of neural and psychophysical responses in the somesthetic system. J. comp. physiol. Psychol., 1960, 53, 42-46.

(Accepted for publication January 26, 1967.) 